\title{
Application of the Vortex Lattice Method to the Three- Dimensional Theory of a Cavitating Propeller
}

\author{
by Hajime Yuasa*, Member
}

\begin{abstract}
Summary
A new theory on a cavitating propeller is proposed, treating the cavitation threedimensionally, and using a lifting surface theory. A numerical method has also been developed based on this theory, appying the vortex lattice method and replacing the cavitation and the blade thickness by source elements, for a cavitating propeller operating in a uniform flow.

To evaluate the present method, the calculations are carried out for a model propeller and compared with other prediction methods and the model test results.

It is confirmed that the present method gives considerable improvements, in comparison with the conventional methods, on the evaluation of the cavity thickness due to the three-dimensional flow, and on the elimination of the instability of the solution caused by the two-dimensional theory.
\end{abstract}

\section{Introduction}

Several theoretical and numerical calculations have been carried out by many authors on cavitating propellers; however, the agreements between the calculated and observed results are not fully satisfactory. Most of the theoretical contributions to this problem are based on the combination of two-dimensional cavitation theory and the angle of attack concept.

For a steady, two-dimensional, thin or slender body, the linearized cavitation problem has been formulated and solved by Tulin ${ }^{1)}$ and Geurst ${ }^{2}$. These previous methods gave the solutions by the analytic function theory in the mapped plane or by the mode function method expressing the singularity distributions in the physical plane. The discrete function method has been developed by Golden ${ }^{3}$ to solve a cavitating two-dimensional hydrofoil.

It was clarified that either the mode function method or the discrete function method has the same characteristics of the solutions, which for instance the cavity becomes very unstable in the transition region between partial and full cavitation ${ }^{4}$.

Uhlman and Persson ${ }^{5}$ tried to improve the instability of the two-dimensional solution by applying the concentrated vortex ahead of the leading edge of a flat plate, and found the stabiliz-

* Akishima Laboratory, Mitsui Engineering and Shipbuilding Co., Ltd. ing effect on the cavity length increased beyond $75 \%$ of chord length.

However, at the outer radial sections of the blade for a cavitating propeller, the traditional method could not yield the solution with a thicker cavity due to the three-dimensional effect.

The natural approach at this point was attempted by Frydenlund and Persson ${ }^{6)}$ describing cavitation on a propeller blade based on the three-dimensional equations but solving them stripwise including three-dimensional induced velocities. For the unsteady cavitating propeller problem, Lee introduced numerical solutions by using a three-dimensional cavity concept, but the cavity length and source distribution were iteratively confined to one chordwise strip by assuming that the cavity extent and source and vortex distributions on the other chordwise strips be known either by an initial approximation or by the most recent solutions on those strips.

These two previous works seem rationally to introduce the three-dimensional effect on a propeller blade, however the process for cavity solution is still limited to the stripwise manner.

In this regard pure three-dimensional cavitation theory and numerical processes are desired to describe cavitation on a propeller blade.

The author attempts to formulate more exactly three-dimensional cavitation theory combined with lifting surface for the steady cavitating propeller problem using the vortex lattice method, and to evaluate the numerical solutions in com- 
parison with experiments, two-dimensional theory and lift equivalent method.

\section{Fundamental Assumption}

The propeller is assumed to operate in an inviscid, unbounded and incompressible fluid, rotating with a constant angular velocity and entailing partial sheet cavitation to a certain extent from the leading edge on the back surface of the propeller blade in a uniform flow.

Fig. 1 shows the co-ordinate system for propeller geometry. The propeller blades, cavity thickness and loading are denoted by the distribution of sources $q(\xi, \eta, \zeta)$ and vortices $\gamma(\xi, \eta, \zeta)$ lying on the mean camber surface of each blade and free vortex sheet.

These sources and vortices will be determined by solving the boundary value problem on the back and face surface of the propeller blade simultaneously.

\section{Boundary Value Problem}

The fluid in which the propeller operates is assumed to be an unbounded, incompressible and inviscid. Therefore, Laplace's equation governs throughout the fluid or the solution must satisfy the boundary conditions specified, which are the normal velocity being zero on the wetted surface of the blade, a constant pressure in the cavity and the closure of the cavity as follows:

\subsection{Wetted Surface Condition}

On a wetted portion of the back and whole face surface of the blade, the kinetic boundary condition that is the normal component of the total fluid velocity should be zero is considered.

Namely, on the portion of the back surface,
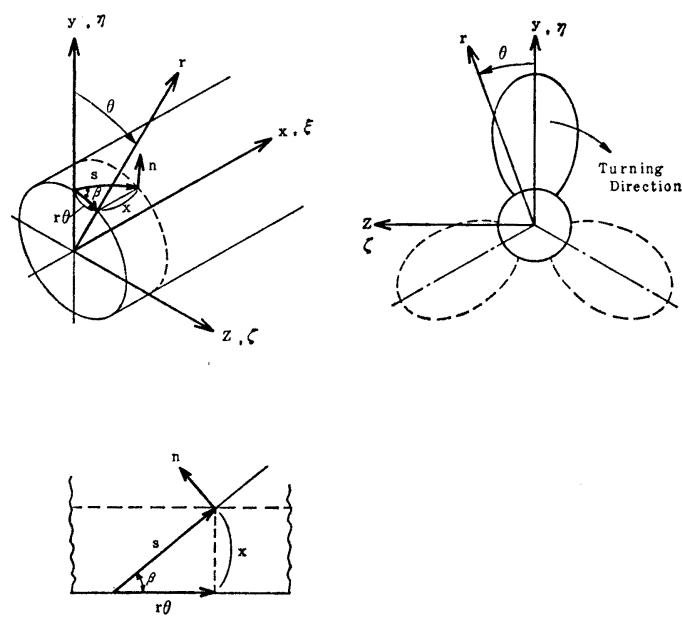

Fig. 1 Co-ordinate system for propeller three-dimensional cavitation theory

$$
\begin{gathered}
\boldsymbol{V}^{G B} \cdot \boldsymbol{n}^{B}+\boldsymbol{V}^{Q B} \cdot \boldsymbol{n}^{B}=-\boldsymbol{W}^{B} \cdot \boldsymbol{n}^{B}, \\
l_{\boldsymbol{r}} \boldsymbol{r}^{c}<l_{\boldsymbol{r}} \leqq 1
\end{gathered}
$$

and on the whole face surface,

$$
\begin{gathered}
V^{G F} \cdot n^{F}+V^{Q F} \cdot n^{F}=-W^{F} \cdot n^{F}, \\
0 \leqq l_{r} \leqq 1
\end{gathered}
$$

where $\boldsymbol{V}^{\boldsymbol{G}}$ and $\boldsymbol{V}^{\mathcal{Q}}$ denote respectively the vectors of induced velocities from the vortex and source distribution with unknown strengths, $\boldsymbol{W}$ is the vector of known inflow velocity, and $\boldsymbol{n}$ is the unit normal vector on the surface specified by the superscripts $B$ and $F$ which indicate Back and Face. $l_{r}$ is the normalized ordinate along the camber line on a blade radial position $r$, where $l_{\boldsymbol{r}}=0$ at the leading edge, $l_{\boldsymbol{r}}=l_{\boldsymbol{r}}{ }^{\mathrm{c}}$ at the cavity end and $l_{r}=1$ at the trailing edge.

\subsection{Cavity Surface Condition}

On the cavity surface the pressure coefficient $-C_{p}$ expressed by Bernoulli's equation is considered to be the same as the cavitation number $\sigma$ defined by the prescribed vapor pressure inside the cavity;

$$
\sigma=-C_{p} \equiv-1+\frac{\left\{\left(\boldsymbol{W}+\boldsymbol{V}^{\tilde{A}}+\boldsymbol{V}^{Q}\right) \boldsymbol{t}\right\}^{2}}{|\boldsymbol{W}|^{2}} .
$$

$\sigma$ is constant on the cavity surface. Hence rewriting expression (3) the following equation is derived,

$$
|\boldsymbol{W}| \sqrt{\sigma+1}=\boldsymbol{W} \cdot \boldsymbol{t}+\boldsymbol{V}^{\boldsymbol{G}} \cdot \boldsymbol{t}+\boldsymbol{V}^{\boldsymbol{Q}} \cdot \boldsymbol{t},
$$

where $\mathbf{t}$ denotes the unit tangential vector on the portion of the back surface (see Fig. 2).

\subsection{Cavity End Condition}

It is assumed that the cavity thickness be zero at the leading edge and be $t_{r}{ }^{c}$ including half the blade thickness at the cavity end $l_{r}{ }^{c}$ which is geometrically shown in Fig. 2. Therefore $t_{r}^{c}$ must satisfy the following equation which is obtained by integrating the gradient of streamline along the camber line;

$$
\begin{aligned}
t_{r^{c}}=\int_{0}^{l_{r} c}\left\{\left(\boldsymbol{W}^{B}+\boldsymbol{V}^{G B}+\boldsymbol{V}^{Q B}\right) \boldsymbol{n}\right. \\
\left.I\left(\boldsymbol{W}^{B}+\boldsymbol{V}^{G B}+\boldsymbol{V}^{Q B}\right) \boldsymbol{t}\right\} d t \\
=\int_{0}^{l_{r^{c}}}\left\{\left(\boldsymbol{W}^{B}+\boldsymbol{V}^{G B}+\boldsymbol{V}^{Q B}\right) \boldsymbol{n} / \boldsymbol{W}^{*} \boldsymbol{t}\right\} d t,
\end{aligned}
$$

where $\boldsymbol{n}$ and $\boldsymbol{t}$ denote the unit normal and tangen-

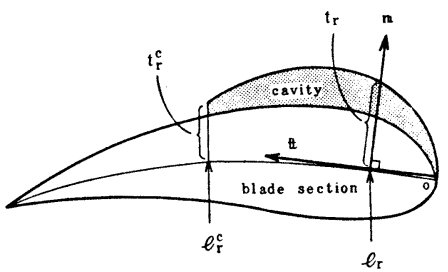

Fig. 2 Definition of cavity end 


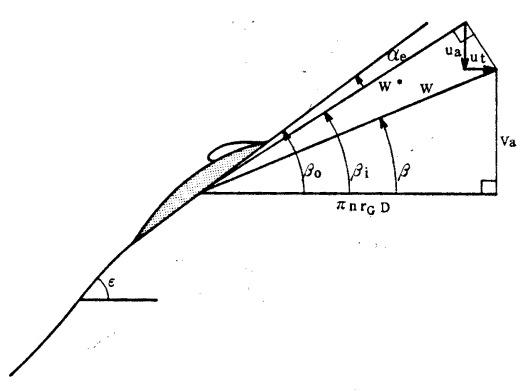

Fig. 3 Velocity diagram

tial vectors on the camber surface in this case.

\section{General Expression of Simultaneous Integral Equation}

As shown in Fig. 3 the vector of known inflow velocity $\boldsymbol{W}$ at a point $\mathrm{P}(x, r, \theta)$ on the $r_{G}$ radial section of the propeller blade can be expressed by using the velocity of uniform flow $V_{a}$ and the propeller turning speed $n$ as follows;

$$
\boldsymbol{W}(x, r, \theta)=\left(V_{a}, 0, \pi n r_{G} D\right),
$$

where $D$ denotes the diameter of the propeller, and the relation between the polar and the Cartesian co-ordinate system is $y=r \cos \theta, z=r$ $\sin \theta$.

The vectors of vortex and source induced velocities are expessed at the same point $\mathrm{P}(x, y$, $z)$,

$$
\begin{aligned}
& \boldsymbol{V}^{G}(x, y, z)=-\frac{1}{4 \pi} \iint_{S+W} \frac{\gamma(\xi, \eta, \zeta) \boldsymbol{l} \times \boldsymbol{R}}{|\boldsymbol{R}|^{3}} d(S+W) \\
& \boldsymbol{V}^{Q}(x, y, z)=-\frac{1}{4 \pi} \iint_{S} \frac{q(\xi, \eta, \zeta) d l \cdot \boldsymbol{R}}{|\boldsymbol{R}|^{3}} d S .
\end{aligned}
$$

Here, the definition of integration is the same as ref. 4. $S$ denotes the camber surface, $W$ the shed wake vortex sheet, $\boldsymbol{R}$ the vector of length between $\mathrm{P}(x, y, z)$ and the singurality point $\mathrm{Q}(\xi$, $\eta, \zeta), \quad l$ the unit vector of the vortices with the arbitrary direction along the camber surface and the shed wake vortex sheet.

Co-ordinate $(\xi, \eta, \zeta)$ are defined as corresponding to the Cartesian co-ordinate system $(x, y, z)$ for the singularity distributions.

Substituting Eqs. (6) to (8) into Eqs. (1) to (5) the following expression of a set of simultaneous integral equations which satisfy the boundary conditions are obtained. These equations for the point $\mathrm{P}(x, y, z)$, include the known values on the right hand side and the unknown $\gamma, q$ and $\sigma$ on the left.

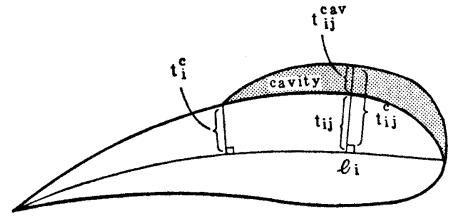

Fig. 4 Definition of cavity thickness

$$
\begin{gathered}
\iint_{S+W} \gamma(\xi, \eta, \zeta) \sum_{p=1}^{Z} \boldsymbol{t}^{B} \cdot \boldsymbol{K}^{G B}(x, y, z ; \xi, \eta, \zeta ; p) d(S+W) \\
+\iint_{S} q(\xi, \eta, \zeta) \sum_{p=1}^{Z} \boldsymbol{t}^{B} \cdot \boldsymbol{K}^{Q B}(x, y, z ; \xi, \eta, \zeta ; p) d S \\
-\left|\boldsymbol{W}^{B}\right| \sqrt{\sigma+1}=\boldsymbol{W}^{B} \cdot \boldsymbol{t}^{B}, \\
0 \leqq l_{\boldsymbol{r}} \leqq l_{\boldsymbol{r}}{ }^{c} \\
\iint_{S+W} \gamma(\xi, \eta, \zeta) \sum_{p=1}^{Z} \boldsymbol{n}^{B} \cdot \boldsymbol{K}^{G B}(x, y, z ; \xi, \eta, \zeta: p) d(S+W) \\
\quad+\iint_{S} q(\xi, \eta, \zeta) \sum_{p=1}^{Z} \boldsymbol{n}^{B} \cdot \boldsymbol{K}^{Q B}(x, y, z ; \xi, \eta, \zeta ; p) d S \\
=W^{B} \cdot \boldsymbol{n}^{B}, \quad(10) \\
l_{\boldsymbol{r}^{c}<l_{\boldsymbol{r}} \leqq 1}
\end{gathered}
$$

$\iint_{S+W} \gamma(\xi, \eta, \zeta) \sum_{p=1}^{Z} \boldsymbol{n}^{F} \cdot \boldsymbol{K}^{G F}(x, y, z ; \xi, \eta, \zeta ; p) d(S+W)$ $+\iint_{S} q(\xi, \eta, \zeta) \sum_{p=1}^{Z} \boldsymbol{n}^{F} \cdot \boldsymbol{K}^{Q F}(x, y, z ; \xi, \eta, \zeta ; p) d S$

$$
=W^{F} \cdot n^{F}
$$

$$
\iint_{S+W} \gamma(\xi, \eta, \zeta) \sum_{p=1}^{Z} \int_{0}^{l_{r} c}\left\{\boldsymbol{n} \cdot \boldsymbol{K}^{G B}(x, y, z ; \xi, \eta, \zeta ; p) /\right.
$$$$
\text { /Wt }\} d t \cdot d(S+W)
$$$$
+\iint_{S} q(\xi, \eta, \zeta) \sum_{p=1}^{Z} \int_{0}^{l^{c}}\left\{\boldsymbol{n} \cdot \boldsymbol{K}^{Q B}(x, y, z ; \xi, \eta, \zeta ; p)\right.
$$$$
\{\boldsymbol{W t}\} d t \cdot d S
$$$$
=-t_{r}{ }^{c}+\int_{0}^{l_{r} c}(\boldsymbol{n} / \boldsymbol{t}) d t
$$

Here, $\boldsymbol{K}^{\boldsymbol{G}}$ and $\boldsymbol{K}^{Q}$ are the kernel functions as follows;

$$
\begin{aligned}
& \boldsymbol{K}^{G}(x, y, z ; \xi, \eta, \zeta ; p)=\frac{1}{4 \pi} \cdot \frac{\boldsymbol{l} \times \boldsymbol{R}}{|\boldsymbol{R}|^{3}} \\
& \boldsymbol{K}^{Q}(x, y, z ; \xi, \eta, \zeta ; p)=\frac{1}{4 \pi} \cdot \frac{\boldsymbol{R}}{|\boldsymbol{R}|^{3}}
\end{aligned}
$$

These Eqs. indicate the difficulties of numerical analysis, due to the doubled singularities in the integral equations.

As shown in Eqs. (9), (10) the cavity length $l_{r}{ }^{c}$ is considered as known and the cavitation number $\sigma$ (= $=-C_{p}$ : kinetic pressure coefficient) is computed. Iterations with respect to the cavity 
length are carried out until $\sigma$ is computed to reach the static pressure coefficient $\sigma_{s}$ given in advance with the prescribed accuracy at each radial section $r . Z$ is the number of blades.

\section{Numerical Analysis}

Reducing these equations by a mode function method to a set of simultaneous algebric equations to solve the problem, one faces the difficulties due to the complex treatments required for the singular integrals. The discrete function method has become popular with respect to the lifting surface problems in recent practical ap-

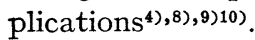

In this chapter the author tries to discretize the problem by using the so called vortex lattice method following the same steps of formulation of the general expression.

\section{1 Arrangement of Singularities}

The camber surface of propeller blades is divided into small rectangular panels the same as the propeller lifting surface.

Dividing the radial (spanwise) direction of the blade into $M$ elements with constant spacing, the radial position of the vortex grid $v_{G}$ is expressed as follows;

$$
\begin{gathered}
v_{G}=v_{h}+\left(1+v_{h}\right) \frac{4 m-3}{4(M-1)+2}, \\
m=1,2, \cdots, M
\end{gathered}
$$

where $v_{h}$ is a hub radius, $v_{G}$ and $v_{h}$ are normalized by the propeller radius. The chordwise position of the vortex grid $S_{G}$ is defined by applying $N$ constant spacing expressed by the normalized ordinate $S$ along the chord;

$$
\begin{gathered}
S_{G}=\frac{n-1}{N}+\frac{1}{4} \cdot \frac{1}{N}, \\
n=1,2, \cdots, N .
\end{gathered}
$$

Including the whole geometrical factors (pitch, rake, offset etc. of the propeller), the vector of specified vortex grid point $\boldsymbol{P}_{i j}^{G}$ on the camber surface of a reference blade is described as follows with respect to the origine of the co-ordinate system;

$$
\boldsymbol{P}_{i j}^{G}=\left(x_{i j}^{G}, y_{i j}^{G}, z_{i j}^{G}\right),
$$

where the subscripts $i$ and $j$ denote the indices of of spanwise and chordwise directions as shown in Fig. 5. The control points $\boldsymbol{P}_{i j}^{C B}$ and $\boldsymbol{P}_{i j}^{C F}$ on the back and face surface of the blade are denoted by using the point $\boldsymbol{P}_{i j}^{C}$ on the camber surface with the unit normal vector $\boldsymbol{n}_{i j}^{C}$ and half the blade thickness $t_{i j}$ at the same point.

$$
\boldsymbol{P}_{i j}^{C B}=\boldsymbol{P}_{i j}^{C}+\boldsymbol{n}_{i j}^{C} t_{i j}
$$

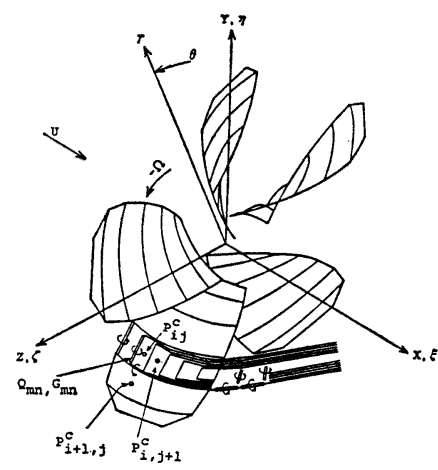

Fig. 5 Schematic view of co-ordinate system and the arrangement of horseshoe vortex system for a propeller

where

$$
\boldsymbol{P}_{i j}^{C F}=\boldsymbol{P}_{i j}^{C}-\boldsymbol{n}_{i j}^{C} t_{i j},
$$

$$
\boldsymbol{P}_{i j}^{C}=\frac{1}{4}\left(\boldsymbol{P}_{i j}^{G}+\boldsymbol{P}_{i+1, j}^{G}+\boldsymbol{P}_{i, j+1}^{G}+\boldsymbol{P}_{i+1, j+1}^{G}\right) .
$$

Regarding the arrangements of singularities, the bound vortex and source elements are placed on the straight line segment between $\boldsymbol{P}_{i j}^{G}$ and $\boldsymbol{P}_{i+1, j}^{G}$ and the trailing vortex element on that between $\boldsymbol{P}_{i j}^{G}$ and $\boldsymbol{P}_{i, j+1}^{G}$. A bound vortex and a couple of trailing elements constitute a horse-shoe vortex. The latter forms a set of helicoidal free vortices toward the infinitive downstream satisfying Kelvin's theorem at each vortex grid point on the blade.

\subsection{Inflow and Induced Velocities}

First, let us discretize the expression (1) to (3). The vector of inflow velocity Eq. (6) can be denoted in the following discrete form;

$$
\boldsymbol{W}_{i j}^{B}=\boldsymbol{W}_{i j}^{F}=\left(V_{a}, 0, \pi n v_{G} D\right) .
$$

The total velocity induced by a vortex distribution is the sum of the influence of all the spanwise and chordwise vortices. Thus the velocity $\boldsymbol{V}_{i j}^{G B}$ at the control point $\boldsymbol{P}_{i j}^{C B}$ on the back surface of the reference blade can be expressed as follows;

$$
\begin{aligned}
\frac{\boldsymbol{V}_{i j}^{G B}}{W}=\sum_{m=1}^{M} \sum_{n=1}^{N} G_{m n} . & \\
& \left\{\boldsymbol{K}_{i j m n}^{G B S}+\sum_{k=N+1}^{\mathbf{V}_{T} T}\left(\boldsymbol{K}_{i j m k}^{G B W}-\boldsymbol{K}_{i j m+1, k}^{G B W}\right)\right\},
\end{aligned}
$$

where $N_{T}-N$ denotes the number of free vortex elements. Here, $W$ is the reference velocity at 0.7 radial position of the blade; $G_{m n}$ is the unknown strength of vortex elements, and detailed algebraic work is shown in Appendix 1. Moreover, by replacing the superscript $B$ by $F, V_{i j}^{G F}$ $W$ can also be obtained for the face surface. 
Referring to the Appendix 2, the source induced velocity can be described for the unknown strength of line source elements for the same control point.

$$
\left.\begin{array}{l}
\frac{\boldsymbol{V}_{i j}^{Q B}}{W}=\sum_{m=1}^{M} \sum_{n=1}^{N} Q_{m n} \boldsymbol{K}_{i j m n}^{Q B}, \\
\frac{\boldsymbol{V}_{i j}^{Q F}}{W}=\sum_{m=1}^{M} \sum_{n=1}^{N} Q_{m n} \boldsymbol{K}_{i j m n}^{Q F} .
\end{array}\right\}
$$

Concerning the cavity surface condition of Eq. (4), the self-induced velocity from the vortex distributions is required for the control point. The following can be denoted from Appendix III.

$$
\begin{aligned}
& \frac{{ }^{b} \boldsymbol{V}_{i j}^{G B}}{W}+\frac{{ }^{t} \boldsymbol{V}_{i j}^{G B}}{W} \\
& =G_{i j} \boldsymbol{H}_{i j}^{G B}+\sum_{k=1}^{j}\left(G_{i-1, k}-G_{i+1, k}\right) \cdot \boldsymbol{H}_{i k}^{G B}, \\
& \frac{{ }^{b} \boldsymbol{V}_{i j}^{G F}}{W}+\frac{{ }^{t} \boldsymbol{V}_{i j}^{G F}}{W} \\
& =-G_{i j} \boldsymbol{H}_{i j}^{G F}-\sum_{k=1}^{j}\left(G_{i-1, k}-G_{i+1, k}\right) \cdot \boldsymbol{H}_{i k}^{G F},
\end{aligned}
$$

where the superscripts $b$ and $t$ mean the contribution from bound and trailing vortex distribution respectively, and

$$
\left.\begin{array}{l}
\boldsymbol{H}_{i j}^{G B}=-\pi \boldsymbol{t}_{i j} /\left(\frac{\Delta c}{R}\right), \\
\boldsymbol{H}_{i j}^{G F}=-\pi \boldsymbol{t}_{i j} /\left(\frac{\Delta c}{R}\right), \\
\boldsymbol{H}_{i k}^{G B}=-\pi \boldsymbol{t}_{i k} /\left(\frac{\Delta s}{R}\right), \\
\boldsymbol{H}_{i k}^{G F}=-\pi \boldsymbol{t}_{i k} /\left(\frac{\Delta s}{R}\right) .
\end{array}\right\}
$$

While for the wetted surface condition of Eqs. (1), (2) the self-induced velocity from the source distribution is required. From Appendix 4 the following can be expressed.

$$
\left.\begin{array}{l}
\frac{\boldsymbol{V}_{i j}^{Q B}}{W}=Q_{i j} \boldsymbol{H}_{i j}^{Q B}, \\
\frac{\boldsymbol{V}_{i j}^{Q F}}{W}=-Q_{i j} \boldsymbol{H}_{i j}^{Q F} .
\end{array}\right\}
$$

Where,

$$
\left.\begin{array}{l}
\boldsymbol{H}_{i j}^{Q B}=-\pi \boldsymbol{n}_{i j} /\left(\frac{\Delta c}{R}\right), \\
\boldsymbol{H}_{i j}^{Q F}=-\pi \boldsymbol{n}_{i j} /\left(\frac{\Delta c}{R}\right),
\end{array}\right\}
$$

$\boldsymbol{t}_{i j}, \boldsymbol{n}_{i j}$ being the unit vectors of tangential and normal directions at the point $\boldsymbol{P}_{i j}^{C}$ on the camber surface on which the singularities are placed.

\subsection{Simultaneous Algebraic Equations}

As the simultaneous integral equations which satisfy the boundary conditions have been derived in the former chapter, the resultant expressions of simultaneous algebraic equation can be obtained to follow the same procedure. Substituting Eqs. (21) to (28) into Eqs. (1) to (5) the discretized simultaneous equations are derived as follows;

$$
\begin{aligned}
& G_{i j} \boldsymbol{H}_{i j}^{G B} \boldsymbol{t}_{i j}^{B}+\sum_{k=1}^{j}\left(G_{i-1, k}-G_{i+1, k}\right) \boldsymbol{H}_{i k}^{G B} \boldsymbol{t}_{i j}^{B} \\
& +\sum_{m=1}^{M} \sum_{n=1}^{N} G_{m n} \sum_{p=1}^{Z}\left\{\boldsymbol{K}_{i j m n}^{G B S}\left(\theta_{\boldsymbol{p}}\right)+\sum_{k=N+1}^{N_{T}}\left(\boldsymbol{K}_{i j m k}^{G B W}\left(\theta_{\boldsymbol{p}}\right)\right.\right. \\
& \left.\left.-\boldsymbol{K}_{i j m+1, k}^{G B W}\left(\theta_{\boldsymbol{p}}\right)\right)\right\} \boldsymbol{t}_{i j}^{B}+Q_{i j} \boldsymbol{H}_{i j}^{G B} \boldsymbol{t}_{i j}^{B} \\
& +\sum_{m=1}^{M} \sum_{n=1}^{N} Q_{m n} \sum_{p=1}^{Z} \boldsymbol{K}_{i j m n}^{Q B}\left(\theta_{\boldsymbol{p}}\right) \boldsymbol{t}_{i j}^{B}-\frac{\left|\boldsymbol{W}_{i j}^{B}\right|}{W} \sqrt{\sigma_{i}+1} \\
& =-\frac{\boldsymbol{W}_{i j}^{B}}{W} \boldsymbol{t}_{i j}^{B} \quad\left(0 \leqq l_{i} \leqq l_{i}{ }^{c}\right) \\
& \sum_{m=1}^{M} \sum_{n=1}^{N} G_{m n} \sum_{p=1}^{Z}\left\{\boldsymbol{K}_{i j m n}^{G B S}\left(\theta_{\boldsymbol{p}}\right)+\sum_{k=N+1}^{N T}\left(\boldsymbol{K}_{i j m k}^{G B W}\left(\theta_{p}\right)\right.\right. \\
& \left.\left.-\boldsymbol{K}_{i j m+1, k}^{G B W}\left(\theta_{p}\right)\right)\right\} \boldsymbol{n}_{i j}^{B}+Q_{i j} \boldsymbol{H}_{i j}^{Q B} \boldsymbol{n}_{i j}^{B} \\
& +\sum_{m=1}^{M} \sum_{n=1}^{N} Q_{m n} \sum_{p=1}^{Z} \boldsymbol{K}_{i j m n}^{Q B}\left(\theta_{p}\right) \boldsymbol{n}_{i j}^{B}+G_{i j} \boldsymbol{H}_{i j}^{G B} \boldsymbol{n}_{i j}^{B} \\
& =-\frac{\boldsymbol{W}_{i j}^{B}}{W} \boldsymbol{n}_{i j}^{B} \quad\left(l_{i}^{c}<l_{i} \leqq 1\right)
\end{aligned}
$$

$$
\begin{aligned}
\sum_{m=1}^{M} & \sum_{n=1}^{N} G_{m n} \sum_{p=1}^{Z}\left\{\boldsymbol{K}_{i j m n}^{G F}\left(\theta_{\boldsymbol{p}}\right)+\sum_{k=N+1}^{N \boldsymbol{T}}\left(\boldsymbol{K}_{i j m k}^{G B W}\left(\theta_{p}\right)\right.\right. \\
& \left.\left.-\boldsymbol{K}_{i j m+1, k}^{G B W}\left(\theta_{\boldsymbol{p}}\right)\right)\right\} \boldsymbol{n}_{i j}^{F}+Q_{i j} \boldsymbol{H}_{i j}^{Q F} \boldsymbol{n}_{i j}^{F} \\
& +\sum_{m=1}^{M} \sum_{n=1}^{N} Q_{m n} \sum_{p=1}^{Z} \boldsymbol{K}_{i j m n}^{Q F}\left(\theta_{\boldsymbol{p}}\right) \boldsymbol{n}_{i j}^{F}+G_{i j} \boldsymbol{H}_{i j}^{G F} \boldsymbol{n}_{i j}^{F} \\
= & -\frac{\boldsymbol{W}_{i j}^{F}}{W} \boldsymbol{n}_{i j}^{F}
\end{aligned}
$$

$$
\begin{aligned}
& \sum_{j=1}^{J} G_{i j} \Delta l_{i j} \boldsymbol{H}_{i j}^{G B} \boldsymbol{n}_{i j} / \boldsymbol{W}_{i j}^{*} \boldsymbol{t}_{i j} \\
& \quad+\sum_{m=1}^{M} \sum_{n=1}^{N} G_{m n} \sum_{p=1}^{Z} \sum_{j=1}^{J} \Delta l_{i j}\left\{\boldsymbol{K}_{i j m n}^{G B S}\left(\theta_{p}\right)\right. \\
& \left.\quad+\sum_{k=N+1}^{N T}\left(\boldsymbol{K}_{i j m k}^{G B W}\left(\theta_{p}\right)-\boldsymbol{K}_{i j m+1, k}^{G B W}\left(\theta_{p}\right)\right)\right\} \boldsymbol{n}_{i j} / \boldsymbol{W}_{i j}^{*} \boldsymbol{t}_{i j} \\
& \quad+\sum_{j=1}^{J} Q_{i j} \Delta l_{i j} \boldsymbol{H}_{i j}^{Q B} \boldsymbol{n}_{i j} / \boldsymbol{W}_{i j}^{*} \boldsymbol{t}_{i j} \\
& \quad+\sum_{m=1}^{M} \sum_{n=1}^{N} Q_{m n} \sum_{p=1}^{Z} \sum_{j=1}^{J} \Delta l_{i j} \boldsymbol{K}_{i j m n}^{Q B}\left(\theta_{p}\right) \boldsymbol{n}_{i j} / \boldsymbol{W}_{i j}^{*} \boldsymbol{t}_{i j} \\
& =t_{i}{ }^{c}-\sum_{j=1}^{J} \Delta l_{i j} \boldsymbol{W}_{i j} \boldsymbol{n}_{i j} / W \boldsymbol{W}_{i j}^{*} \boldsymbol{t}_{i j}
\end{aligned}
$$

Where $J$ is the corresponding $j$ of the unknown ordinate $l_{i}{ }^{c}$ along the chord where the cavity terminates on the $i$-th radial panel of the reference blade. $Z$ is the number of blades and $\theta_{p}$ is the angle position of each blade.

Therefore Eqs. (29) through (32) lead to a set of simultaneous linear equations for the unknown 
vortices $G_{m n}$, sources $Q_{m n}$ and cavitation numbers $\sigma_{i}$, if initially assuming the cavity length at spanwise section $r_{i}$.

Here $\boldsymbol{W}_{i j}^{*}$ in Eq. (32) is corresponding to $\boldsymbol{W}^{*}$ in Eq. (5) and $\boldsymbol{W}^{B}$ may be given as its value at the initial step of the computation.

\subsection{Cavity Shape}

Applying the solutions obtained from the simultaneous equations, the cavity shape can be calculated from Eq. (5). This can be rewritten as the following discretized form at $l_{i j}$ of the arbitrary chordwise ordinate which yields the height from the camber surface summing up the cavity and half the blade thickness.

$$
\begin{aligned}
t_{i J}{ }_{j}= & \sum_{j=1}^{J}\left\{\Delta l_{i j} \boldsymbol{W}_{i j} \boldsymbol{n}_{i j}+\Delta l_{i j} G_{i j} \boldsymbol{H}_{i j}^{G B} \boldsymbol{n}_{i j}\right. \\
& +\sum_{m=1}^{M} \sum_{n=1}^{N} G_{m n} \sum_{p=1}^{Z} \Delta l_{i j}\left\{\boldsymbol{K}_{i j m n}^{G B S}\left(\theta_{\boldsymbol{p}}\right)\right) \\
& \left.+\sum_{k=N+1}^{N T}\left(\boldsymbol{K}_{i j m k}^{G B W W}\left(\theta_{\boldsymbol{p}}\right)-\boldsymbol{K}_{i j m+1, k}^{G B W}\left(\theta_{\boldsymbol{p}}\right)\right)\right\} \boldsymbol{n}_{i j} \\
& +\Delta l_{i j} Q_{i j} \boldsymbol{H}_{i j}^{Q B} \boldsymbol{n}_{i j} \\
& +\sum_{m=1}^{M} \sum_{n=1}^{N} Q_{m n} \sum_{p=1}^{Z} \Delta l_{i j}\left\{\boldsymbol{K}_{i j m n}^{O B}\left(\theta_{\boldsymbol{p}}\right) \boldsymbol{n}_{i j}\right\} / \boldsymbol{W}_{i j}^{*} \boldsymbol{t}_{i j},
\end{aligned}
$$

where $J$ is chordwise index corresponding to the ordinate $l_{i}$ as shown in Fig. 4.

The cavity thickness $t_{i j}^{\text {cav }}$ can be obtained at $l_{i}$ as follows;

$$
t_{i J}^{\mathrm{car}}=t_{i J}^{c}-t_{i J} .
$$

Here $t_{i j}$ is half the blade thickness.

\section{Results of Computation}

In order to confirm the validity of the present method, the computation has been carried out for a Japanese standard propeller model (Table 1) with partially cavitating condition operating in a uniform flow.

The number of panels on the blade surface has been applied as follows:
Table 1 Principal particulars of model propeller

\begin{tabular}{|l|l|}
\hline Model Propeller & MAU 3-50 \\
\hline Dia. (M) & 0.250 \\
\hline Pitch Ratio & 1.000 \\
\hline Exp. Area Ratio & 0.500 \\
\hline Number of Blades & 3 \\
\hline
\end{tabular}

\section{1 elements along the chord}

6 elements along the span (equal spacing), where the chord length is equally divided into 10 and, moreover, the nearest panel to the leading edge is divided equally.

It is required to increase the number of panels if one intends to improve the accuracy of the computation. The number of panels described above has been determined by examining the balance between the accuracy and the cost of the computation for the present purposes.

Each element of bound vortex and line source has been placed on the quarter chord line and the control point on the midspan of three-quarter chord position on each panel according to James $^{12)}$.

For the propeller wake geometry, the deformed model has been adopted which was introduced by the author ${ }^{11)}$ combining the contraction and the classical wake models with the same pitch as the local blade pitch distributions. This is suggested to predict both the propeller performance and the slipstream over the wide range of propeller loading with sufficient accuracy.

Thus defining the arrangements of panel division and propeller wake geometry, the simultaneous Eqs. (29) to (32) can be solved by setting up an operating condition of the propeller.

\subsection{Influence of Blade Loading}

In order to confirm the tendency of the solu-

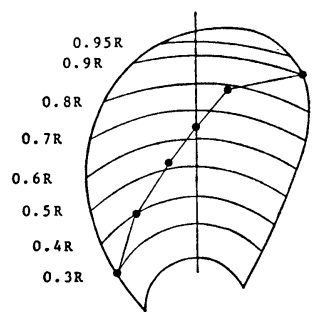

Trial - 1

Na $1-0$
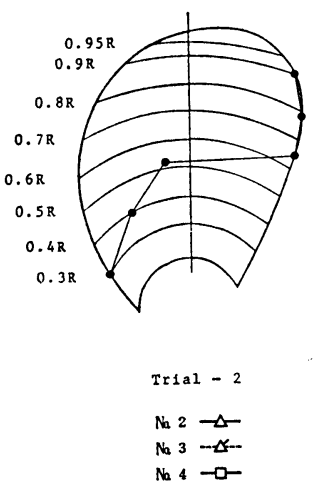

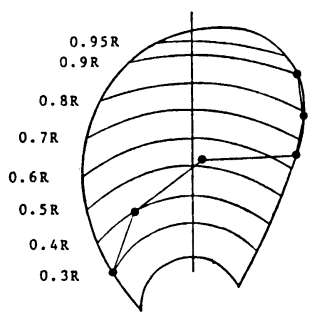

$\operatorname{Tr} 1 \mathrm{a} 1-3$

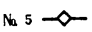

Fig. 6 Cavitation patterns given for the trial calculations (MAU 3-50, $H / D=1.0$ ) 


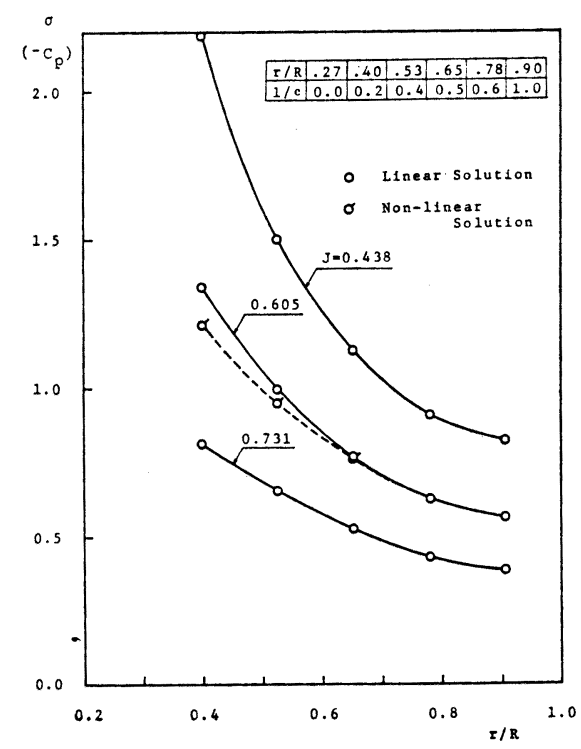

Fig. 7 Solutions of cavitation number $\sigma$ for various loading conditions in case of Trial-1

tion toward the influence of blade loading, a simple pattern of sheet cavitation has been assumed (Trial-1 in Fig. 6) with the cavity end being zero.

Fig. 7 shows the solutions of the cavitation number $\sigma$ at three advance ratios. The results that the solution $\sigma$ becomes higher in the case of fixed cavity length when the propeller loading increases, indicating the same characteristics of the actual phenomena. The description "NonLinear Solution" in the figure means the results of iterative computation with respect to the denominator in Eq. (5). The Non-Linear Solution agrees with the Linear Solution in the region from the midspan to the tip of the blade.

Fig. 8 displays the cavity shape by putting the solutions of vortices and sources into Eq. (33).

For the cavity length larger than $75 \% \mathrm{C}$ (of the chord length) the solutions are also obtained with no sign of instability as in the solution of the basic two-dimensional cavitation theory. The results of cavity thickness show an optimal tendency from the fact that it becomes larger due to the increment of propeller loading.

The author is especially impressed that the cavity thickness around the tip region or at $0.9 R$ reaches large enough, i.e. 10 to $25 \% \mathrm{C}$ as shown in Fig. 8 , because he introduced a three-dimensional correction factor to the solution of the twodimensional cavitation theory for that region of propeller blade cavitation in the previous paper $^{4)}$. The correction factor, determined by

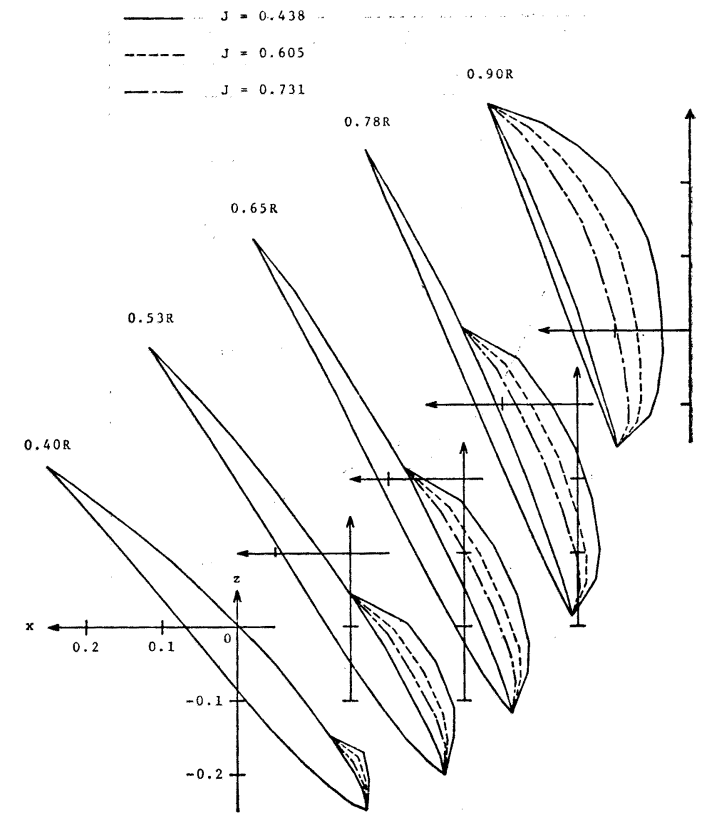

Fig. 8 Cavity shapes obtained for Trial-1 with closed cavity ends (No. 1)

investigating the full-scale measurements of cavity thickness, was multiplying by "five" the two-dimensional solution. The cavity thickness obtained by the two-dimensional theory is 2 to $3 \% \mathrm{C}$ at that region in general. Since the present solution gains "five to ten" times that, it might be said that the prescribed correction factor "five" is correct.

\subsection{Iteration for Cavity Shape}

The computation has to be repeated to determine the cavity shape until the solution $\sigma(v)$ reaches the value of given cavitation number $\sigma_{s}(\gamma)$ at each radial section of a propeller blade. According to the comparison of both mentioned above;

$\sigma(v)>\sigma_{s}(v)$ : the cavity extent being increased along the chordwise panel at radial position $r$, or the thickness of the cavity end being expanded in the case of full cavity;

$\sigma(v)<\sigma_{s}(v)$ : reverse of that described above. Thus, the iterative computation is to be carried out.

Fig. 9 shows the results of a that calculation in which the cavity extent and its closure condition are varied at each radial section in order to let the solution $\sigma(r)$ approach $\sigma_{s}(r)$ of the model experiment. As denoted in the upper table in Fig. 9, one can investigate the solutions approaching the given values by varying the combination of the cavity extent, its closu吕 condition and 


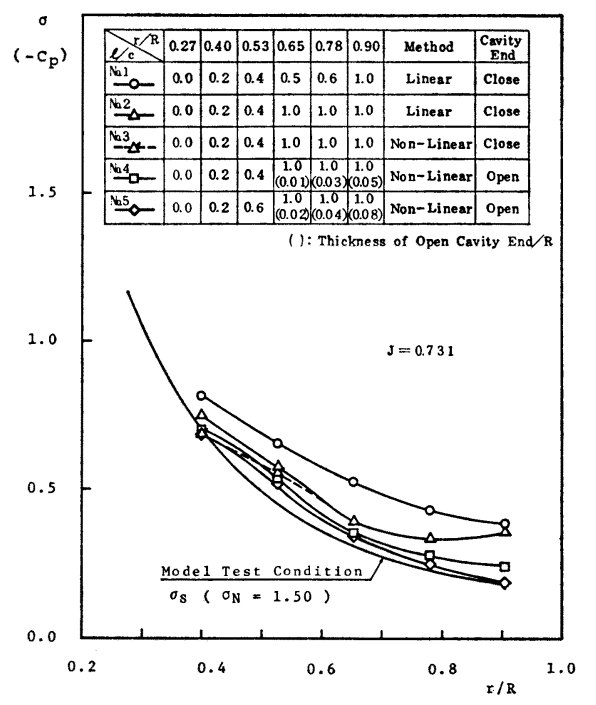

Fig. 9 Solutions of cavitatiin number $\sigma$ for the various cavitation patterns (Trial$1,2, \& 3)$ and cavity end conditions (No. $1 \sim 5$ )

Linear or Nonlinear regarding each case of No. 1 to 5 at the same loading condition.

In the case of No. 5 the cavitation pattern cor-

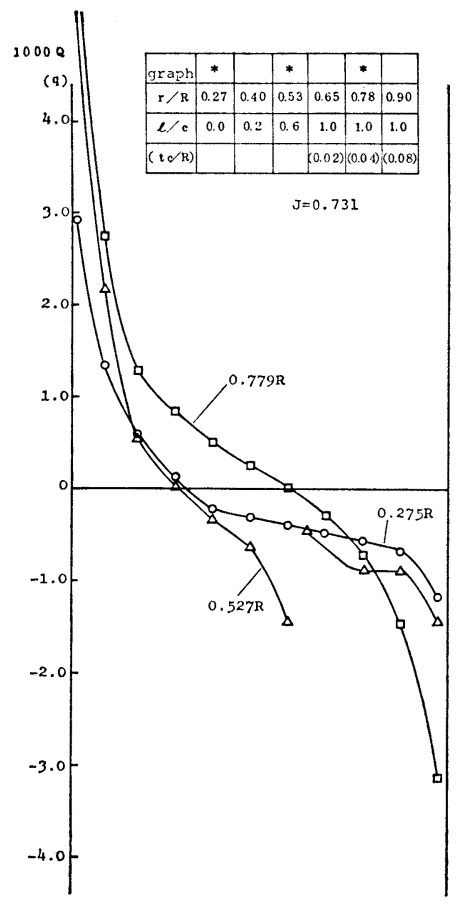

(a) responds to the trial-3 in Fig. 6 which has little larger extent and wider thickness of the cavity end at the sections with full cavity than that of No. 4. The solution of No. 5 comes so close to $\sigma_{s}(v)$ that it might be regarded as the approximate converged solution.

Figs. 10 and 11 are the solutions of sources $q$ and vortices $\gamma$ in the case of No. 5. With respect to the blade section with partial cavity, the distributions of $q$ and $\gamma$ show the discontinuity at the cavity ends, which is the common characteristic of two-dimensional cavitation theory. Moreover, the distribution of $\gamma$ on the blade section with full cavity can considerably be seen flat.

Fig. 12 displays the results of cavity shape in the case of No. 5. For the blade sections near the root with large thickness, for example $0.53 R$, the maximum thickness of the partial cavity is 1 to $2 \% \mathrm{C}$ even if the cavity is assumed to separate at the leading edge. The corresponding solution of two-dimensional theory is 3 to $4 \% \mathrm{C}$, but the actual measurement is about $1 \% \mathrm{C}$ quoted from ref. 4. The maximum thickness of the partial cavity can be reduced in the case of two-dimensional theory by assuming that the cavity starts at the minimum pressure point which locates downstream near the leading edge, and its results

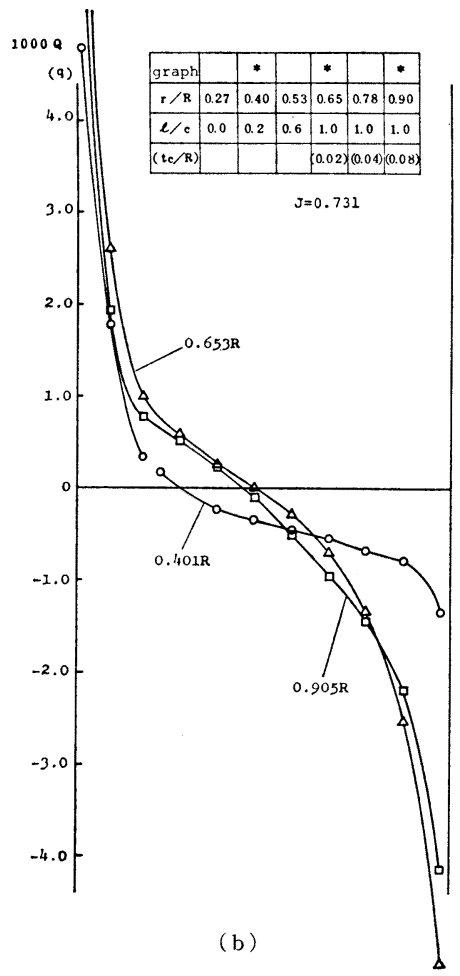

Fig. 10 Source strength obtained for Trial-3 with open cavity ends (No. 5) 

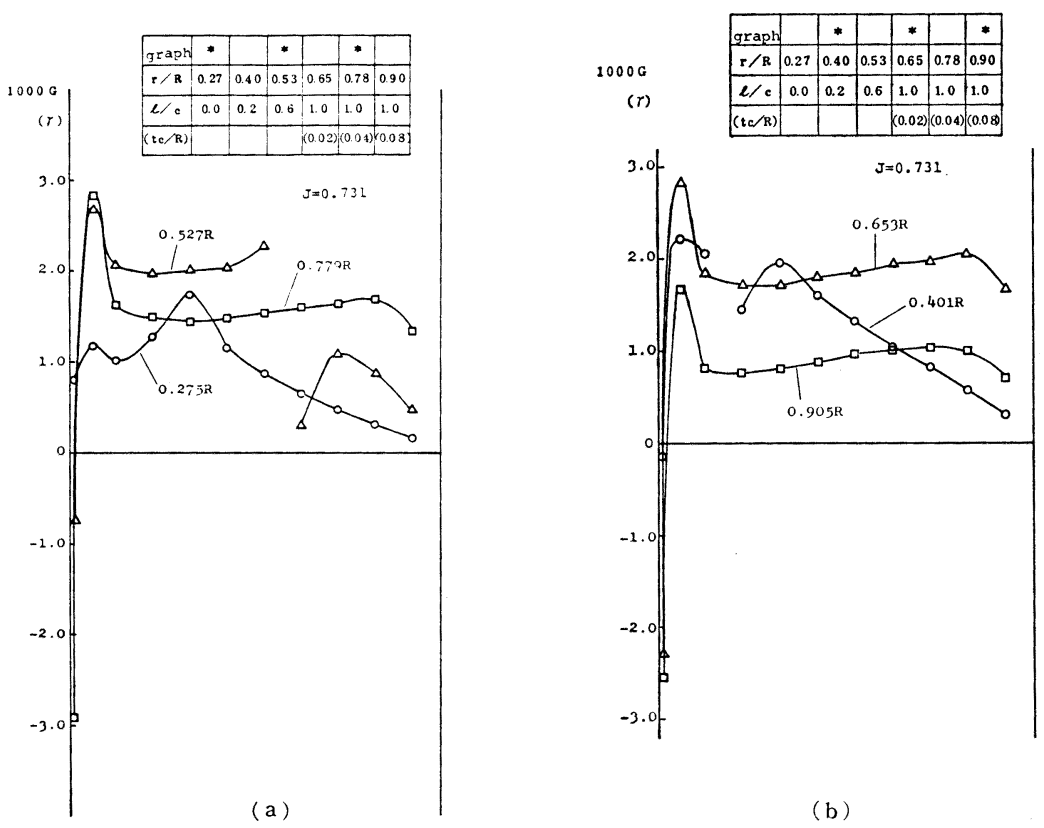

Fig. 11 Vortex strength obtained for Trial-3 with open cavity ends (No. 5)

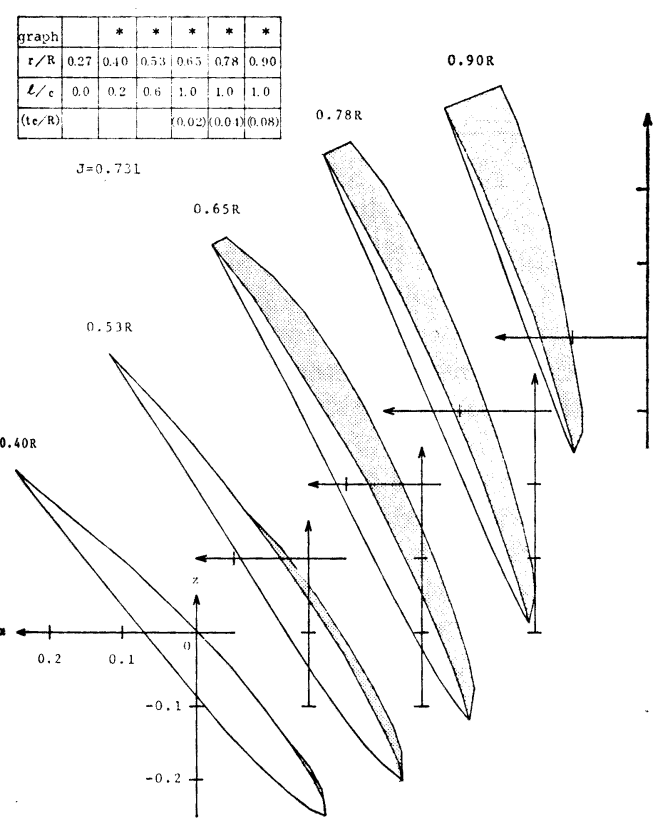

Fig. 12 Cavity shapes obtained for Trial3 with open cavity ends (No. 5)

show good agreement with the measurement ${ }^{13)}$. - From these considerations, the solution of the present three-dimensional theory can yield good results for the maximum thickness of the partial cavity even if the cavity is assumed to separate at the leading edge.

In the present method the thickness of the full cavity end is supposed to vary in order to obtain the converged solution. However it must actually become a super-cavity stretching its tail longer when the cavitation number decreases.

Accordingly, one should expand this method to simulate such an actual phenomenon as threedimensional cavitation including the supercavity, which requires the increase of the unknown values and computing time in proportion to the cavity length.

In any case it is considered that the present method is enough to discuss the characteristics of the three-dimensional solution for the sake of simplification and time saving. With respect to the convergence of the solution, the present method shows the following relations by inquiring into the cases of No. 1 to No. 5 ;

$$
\begin{aligned}
& \text { cavity length } l_{\boldsymbol{r}}{ }^{c} \rightarrow \text { increase } \quad \begin{array}{c}
\text { solution } \\
\sigma(r) \rightarrow \text { decrease }
\end{array} \\
& \rightarrow \text { decrease } \rightarrow \text { increase }
\end{aligned}
$$

thickness of the full cavity end

propeller loading

$$
\begin{aligned}
t_{\boldsymbol{r}}{ }^{c} & \rightarrow \text { increase } & \sigma(v) & \rightarrow \text { decrease } \\
& \rightarrow \text { decrease } & & \rightarrow \text { increase } \\
& & & \\
K_{T} & \rightarrow \text { increase } & \sigma(v) & \rightarrow \text { decrease } \\
& \rightarrow \text { decrease } & & \rightarrow \text { increase }
\end{aligned}
$$

These tendencies guarantee the convergence of the solution by the present method, since the 

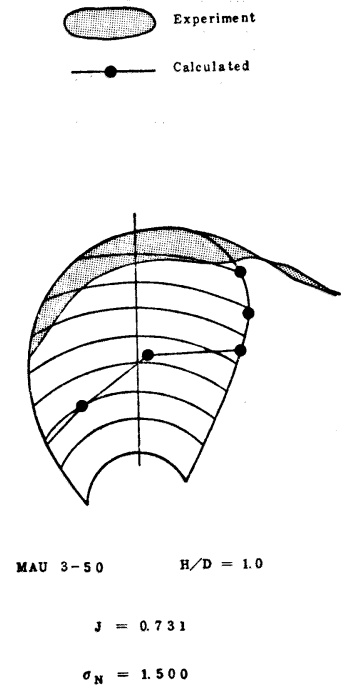

Fig. 13 Comparison of cavitation patterns between the experiment and Trial-3 corresponding to the converged solution by the present three-dimensional calculation (No. 5)

parameters described above vary to adapt themselves to the tendency of the actual phenomena. That is to say, one can let $\sigma(v)$ agree with $\sigma_{s}(\gamma)$ by controlling the parameters $l_{\boldsymbol{r}}{ }^{c}$ or $t_{\boldsymbol{r}}{ }^{c}$ at $l_{\boldsymbol{r}}{ }^{c}=1$.

\subsection{Comparison with Experiment}

Fig. 13 shows the comparison between the model experiment and the present solution of the case of No. 5 in a uniform flow. The experiment discloses the narrow sheet cavitation along the leading edge, while the solution presents the larger extent of sheet cavitation with the full cavity in the region from $0.65 R$ through the tip.

Though the convergence of the present solution has been confirmed by referring to the actual phenomena in the former section, the calculated extent of cavity by the present method could not agree with the model experiment. In order to investigate the disagreement of the present solution with the model experiment, this has been compared with the conventional liftequivalent method and the modified twodimensional cavitation theory quoted from ref. 4 .

Fig. 14 shows the pressure distributions on the propeller blade at the wetted surface condition with three kinds of cavitation number $\sigma_{N}(=P /$ $\left.\frac{1}{2} \rho_{n}^{2} D^{2}\right)$ including $\sigma_{N}=1.50$ in the case of No. 5 for each radial section. For the three conditions of $\sigma_{N}$ the calculated results by the conventional method are shown in Fig. 15 compared with the present method. Here, the two-dimensional calculation has been carried out by using the
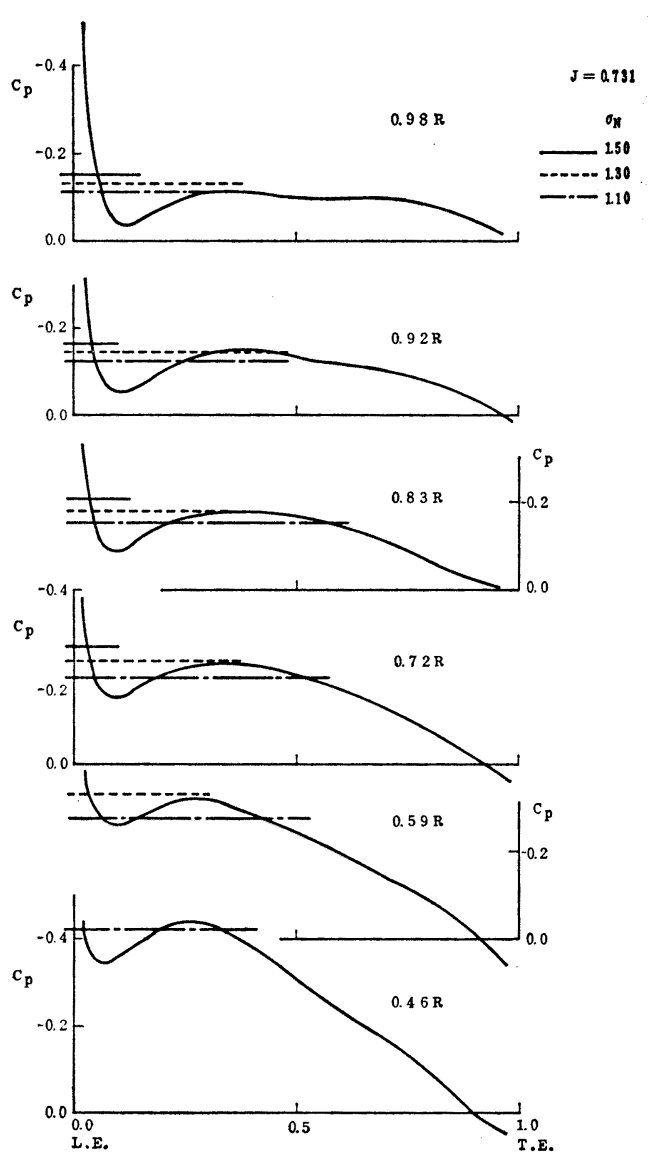

Fig. 14 Pressure distributions for lift-equivalent method (MAU 3-50, $H / D=$ 1.0)

interpolation method referred to in ref. 4 for the case that the cavity length increases beyond $75 \% \mathrm{C}$.

Fig. 15 shows the good agreement between the present method and the two-dimensional method, but the result of the lift-equivalent method is rather close to the experiment. The reason for the difference between the present method and the experiment is thought to be caused by $\sigma_{\Delta}$ being similar to the pressure distribution downstream the hollowed pressure near the leading edge on the blade. Accordingly, it should be noted that the observed sheet cavitation in the negative pressure region at the leading edge terminates around the hollowed pressure point of 10 to $20 \% \mathrm{C}$ while the present result extends the cavity length to the trailing edge because of the slight difference between $\sigma_{N}$ and the pressure distribution.

Another reason could be that the sheet cavitation is shortened by the effect of the laminar boundary layer on the blade surface due to the 


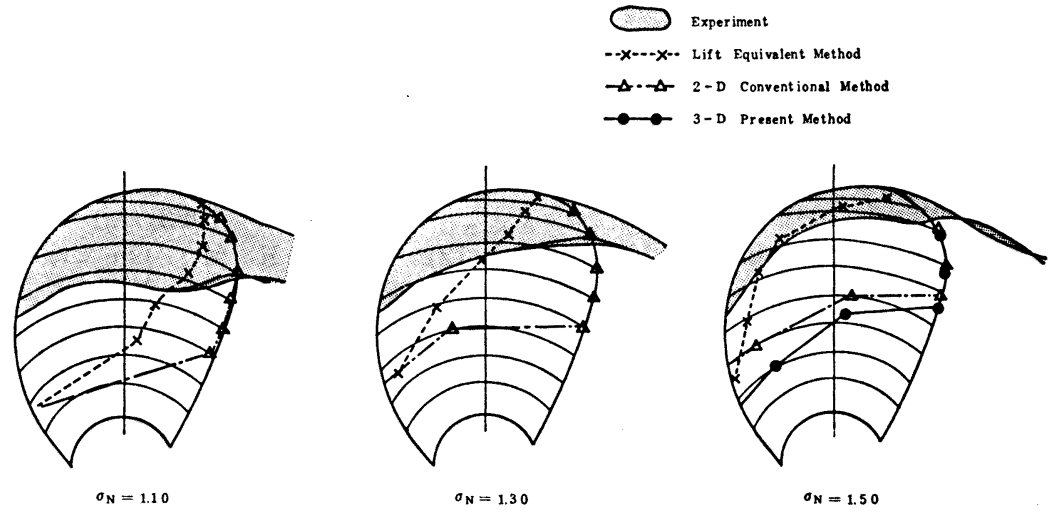

Fig. 15 Comparison of cavitation patterns between the experiment, liftequivalent method, $2-D$ cavity conventional method and $3-D$ cavity present method (MAU 3-50, $H / D=1.0$ )

model scale, because it was observed that the lower radial position of the sheet cavitation had no variation when $\sigma_{N}$ decreased.

Therefore adopting the leading edge roughness as a turbulent stimulator, the expriment will be close to the present results. This is thought to be a proper problem with respect to the technique for model testing ${ }^{14)}$. On reflection, the full scale observation may be rather close to the present method.

\section{Concluding Remarks}

A new theory on a cavitating propeller is proposed, treating the cavitation three-dimensionally, and using a lifting surface theory. A numerical method has also been developed based on this theory, applying the vortex lattice method and replacing the cavitation and the blade thickness by source elements, for a cavitating propeller operating in a uniform flow.

Based on the proposed theory, the following approaches are attempted.

a) Discrete panels, vortex and source elements are placed on the camber surface to denote the blade loading and thickness effect including threedimensional cavitation, while the boundary conditions are to be satisfied at the control points on both back and face surface of propeller blades.

b) With respect to the self-induced velocity due to vortex distributions, the contributions from the chordwise elements are exactly considered together with that of the spanwise elements.

c) The induced velocities by the whole vortices and sources are taken into account on the cavity closure condition without simplification.

d) To evaluate the present method, the calculations are carried out for a model propeller and compared with other prediction methods and the model test results.

The advantages of this method could be summerized as follows:

(1) At a certain loading condition, the solution $\sigma(v)$ can reach the value of given cavitation number $\sigma_{s}(r)$ by the iterative computation controlling the cavity length and the thickness of cavity end.

(2) The present method indicates no sign of the $75 \%$ chord length break down which is wellknown shortcoming in case of the two-dimensional cavitation theory.

(3) With respect to the cavity thickness around the tip region, three-dimensional effect can naturally be taken into account, and the results give the cavity shapes that are sufficiently close to the ones observed in full-scale measurements.

(4) The results show the possibilities to eliminate the overestimate for a thick profile around the blade root which is generally the case by the two-dimensional theory.

(5) As for the cavitation extent, the present method gives a good agreement with other prediction method by the modified two-dimensional theory, but shows disagreements with the model test results and also with the results by the lift-equivalent method. This insufficient agreement of cavity extent between the present method and the model test may be caused by the effect of laminar boundary layer on the blade surface in the model test.

It is confirmed that the present method gives considerable improvements, in comparison with the conventional method, on the cavity thickness due to the three-dimensional flow. By examining the detailed characteristics of the present solution, it is however confirmed that the numerical 
computation is exactly performed. Thus, the discrete function method as vortex lattice concept is a powerful technique to solve such a complex problem which seems very difficult to solve by applying mode function method etc.

\section{Acknowledgement}

The author is grateful to Professor H. Kato of Tokyo University for his guidance and valuable suggestions throughout the work.

He wishes to express his sincere gratitude to Professor Yamanouchi of Nippon University with the additional post of the advicer of Mitsui E. \& S., Mr. Y. Sugimura, General Manager and Mr. M. Abe, Manager, Mr. N. Ishii and Mr. H. Kamiirisa of Akishima Laboratory of Mitsui $\mathrm{E}$. $\&$ S. for their cooperation in carrying out the present study.

\section{References}

1) Tulin, M. P.: Supercavitating FlowsSmall Perturbation Theory, J. Ship Research, Vol. 7, No. 3 Jan. (1964).

2) Geurst, J. A.: Linearized Theory of TwoDimensional Cavity Flows, Thesis, Delft Technical Institute, The Netherlands (1961).

3) Golden, D. W.: A Numerical Method for Two-Dimensional, Cavitating, Lifting Flow, MIT, Dept. of Ocean Eng., Rept. No. 81512-1 (1975).

4) Yuasa, H. et al.: Practical Applications of the Discrete Vortex Element Method for Calculation of Propeller Induced Excitation Forces, 13th Symposium on Naval Hydrodynamics, Tokyo, Oct. (1980).

5) Uhlman, J. S. and Persson, B.: Influence of a Leading Edge Vortex on the Partial Cavitation on a Flat Plate, to be published.

6) Frydenlund, O. and Persson, B.: Application of a Discrete Vortex, Source Distribution Model to Propeller Cavitation, J. Ship Research, Vol. 25, No. 24 (1981).

7) Lee, C.-S.: Prediction of the Transient Cavitation on Marine Propellers by Numerical Lifting-Surface Theory, 13th Symposium on Naval Hydrodynamics, Tokyo, Oct. (1980).

8) Kerwin, J. E. and Lee, C.-S.: Prediction of Steady and Unsteady Marine Propeller Performance by Numerical Lifting-Surface Theory, Trans. SNAME, Vol. 86 (1978).

9) Szantyr, J.: A Computer Program for Calculation of Cavitation Extent, Excitation Forces for a Propeller Operating in Non-Uniform Velocity Field, I.S.P., Vol. 26, No. 296 (1979).

10) Ikehata, M. et al.: The Analysis of Un- steady Characteristics of Marine Propeller in Harmonic Wake by Vortex Lattice Lifting-Surface Model, J. Soc. Naval Arch. Japan, Vol. 153 (1983).

11) Yuasa, $H$. et al.: A Consideration on Propeller Slipstream, Mitsui Zosen Technical Review (1983)

12) James, R. M.: On the Remarkable Accuracy on the Vortex Lattice Method, Computer Methods in Applied Mechanics and Engineering, 1 (1972).

13) Nishiyama, T. and Ito, J.: Fundamental Equation and Its Analysis of the Flow Field for Partially Cavitating Hydrofoil by a Singularity Method, Part 5, Isolated Profile with Large Camber and Thickness, J. of the Soc. Mech. Japan (1980).

14) Ishii, N. et al.: Model Testing of Propeller Cavitation by Roughening the Leading Edge of Blades, J. Soc. Naval Arch. Japan, Vol. 153 (1983).

\section{Appendix 1 Vortex Induced Velocity}

The induced velocity $\boldsymbol{V}_{i j}^{G}\left(\overline{\boldsymbol{X}}_{\boldsymbol{k}} \overline{\boldsymbol{X}}_{k+1}\right)$ at a control point $P_{i j}$ due to a vortex element $\overline{\boldsymbol{X}}_{k} \overline{\boldsymbol{X}}_{k+1}$ on a set of horse-shoe vortex which is constituted from $m n$-th bound vortex and a couple of trailing vortices can be expressed by the law of BiotSavart as follows:

$$
\boldsymbol{V}_{i j}^{G}\left(\overline{\boldsymbol{X}}_{k} \boldsymbol{X}_{k+1}\right) \equiv \boldsymbol{V}_{i j m n}^{G}=\frac{\Gamma_{m n}}{4 \pi} \int_{\boldsymbol{X}_{k}}^{\boldsymbol{X}_{k+1}} \frac{d \boldsymbol{l} \times \boldsymbol{D}}{|\boldsymbol{D}|^{3}},
$$

where the vortex strength per unit length $\Gamma_{m n}$ is constant on the horse-shoe vortex, $d \boldsymbol{l}$ the differential vector of the element, $\boldsymbol{D}$ the vector of the distance between $d \boldsymbol{l}$ and the control point. The subscripts $m$ and $n$ demote the indices of spanwise and chordwise directions on the propeller blade.

Dividing Eq. (A1.1) by the reference velocity $W$ the following expression is derived:

$$
\boldsymbol{V}_{i j m n}^{G} / W=G_{m n} \boldsymbol{K}_{i j m n}^{G}
$$

where supposing $R$ being the radius of the propeller,

$$
\begin{aligned}
& G_{m n}=\Gamma_{m n} / 2 \pi W R, \\
& \boldsymbol{K}_{i j m n}^{G}=\frac{1}{2} R \int_{\boldsymbol{X}_{k}}^{\boldsymbol{X}_{k+1}} \frac{d \boldsymbol{l} \times \boldsymbol{D}}{|\boldsymbol{D}|^{3}} .
\end{aligned}
$$

Therefore, the induced velocity $\boldsymbol{V}_{i j}^{G}$ due to the whole horseshoe vortices denoting propeller blades and its free vortex sheet can be described as follows:

$$
\begin{aligned}
\boldsymbol{V}_{i j}^{G} / W= & \sum_{m=1}^{M} \sum_{n=1}^{N} G_{m n}\left\{\boldsymbol{K}_{i j m n}^{G S}\right. \\
& +\sum_{k=N+1}^{N T}\left(\boldsymbol{K}_{i j m k}^{G W}-\boldsymbol{K}_{i j m+1, k)}^{G W}\right\}
\end{aligned}
$$


where,

$$
\boldsymbol{K}_{i j m n}^{G S}=\boldsymbol{K}_{i j m n}^{G S \operatorname{span}}+\sum_{k=n}^{N}\left(\boldsymbol{K}_{i j m k}^{G S \operatorname{chord}}-\boldsymbol{K}_{i j m+1, k}^{G S \operatorname{chord}}\right)
$$

Hence, the superscripts $S$ and $W$ mean blade surface and free wake vortex sheet, $M, N$ and $N_{T}$ $-N$ denote the numbers of spanwise, chordwise and free vortex elements respectively.

\section{Appendix 2 Source Induced Velocity}

The source strength per unit length $\sigma_{m n}$ is defined to be constant along $m n$-th spanwise element. Supposing the co-ordinate position of its both ends $\boldsymbol{X}_{m n}$ and $\boldsymbol{X}_{m+1, n}$, the induced velocity $\boldsymbol{V}_{i j m n}^{\ell}$ at the control point $P_{i j}$ due to the source element $\overline{\boldsymbol{X}}_{m n} \overline{\boldsymbol{X}}_{m+1, n}$ as follows:

$$
\boldsymbol{V}_{i j m n}^{Q_{m}}=\frac{\sigma_{m n}}{4 \pi} \int_{\boldsymbol{X}_{m n}}^{\boldsymbol{X}_{m+1, n}} \operatorname{grad}\left(\frac{1}{|\boldsymbol{D}|}\right) d l
$$

Non-dimensionalizing Eq. (AII.1)

$$
\boldsymbol{V}_{i j m n}^{o} / W=Q_{m n} \boldsymbol{K}_{i j m n}^{o}
$$

is expressed where

$$
\begin{aligned}
& Q_{m n}=\sigma_{m n} / 2 \pi W R, \\
& \boldsymbol{K}_{i j m n}^{Q}=\frac{1}{2} R \int_{\boldsymbol{X}_{m n}}^{\boldsymbol{X}_{m+1, n}} \operatorname{grad}\left(\frac{1}{|\boldsymbol{D}|}\right) d l .
\end{aligned}
$$

The contribution from the whole source elements can therefore be denoted as follows:

$$
\boldsymbol{V}_{i j}^{Q} / W=\sum_{m=1}^{M} \sum_{n=1}^{N} Q_{m n} \boldsymbol{K}_{i j m n}^{O}
$$

\section{Appendix 3 Self-Induced Velocity due to Vortex Distribution}

Discrete vortex elements are regarded as the concentration of the vortex distribution density $\gamma$. The relation between the discretized vortex strength per unit length $\Gamma_{i j}$ and $\gamma$ on $i j$-th panel of a propeller blade can be derived as follows.

$$
\begin{aligned}
& { }^{b} \gamma_{i j}=\Gamma_{i j} / \Delta c \\
& { }^{t} \gamma_{i j}=\frac{1}{2} \sum_{k=1}^{i}\left(\Gamma_{i-1, k}-\Gamma_{i+1, k}\right) / \Delta s
\end{aligned}
$$

where the superscripts $b$ and $t$ denote bound and trailing, $\Delta c$ and $\Delta s$ the mean length along chord and span of the panel. The vortex distribution densities ${ }^{b} \gamma_{i j}$ and ${ }^{t} \gamma_{i j}$ are supporsed to be constantly distributed on $i j$-th panel.

The self-induced velocities due to the vortex distribution can be expressed at the control point $P i j$ as follows :

$$
\begin{aligned}
{ }^{b} V_{i j}^{G} / W & =-\frac{1}{2}{ }^{b} \gamma_{i j} / W \equiv-\pi G_{i j} /(\Delta c / R) \\
{ }^{t} V_{i j}^{G} / W & =-\frac{1}{2}{ }^{t} \gamma_{i j} / W \\
& \equiv-\pi \sum_{k=1}^{j}\left(G_{i-1, k}-G_{i+1, k}\right) /(\Delta s / R)
\end{aligned}
$$

Here, ${ }^{b} V_{i j}^{G}$ and ${ }^{t} V_{i j}^{G}$ denote the induced velocities due to bound and trailing vortex distribution densities, and the values $G_{0 k}$ and $G_{M+1, k}$ of the root and tip panels are defined as follows:

$$
G_{0 k}=G_{1 k}, \quad G_{M+1, k}=G_{M, k}
$$

The expressions (A3.3) and (A3.4) yield the tangential velocities to the camber surface.

\section{Appendix 4 Self-Induced Velocity due to Source Distribution}

Referring to the case of vortex the source distribution density $q_{i j}$ is expressed by using the discretized source strength per unit length $\sigma_{i j}$ as follows:

$$
q_{i j}=\sigma_{i j} / \Delta c
$$

The self-induced velocity due to the vortex distribution can be described as the following expression at the control point $P_{i j}$.

$$
V_{i j}^{Q} / W=-\frac{1}{2} q_{i j} / W \equiv-\pi Q_{i j} /(\Delta c / R)
$$

This yields the normal velocity to the camber surface. 